\title{
Gauge symmetry and constraints structure for topologically massive AdS gravity: a symplectic viewpoint
}

\author{
Omar Rodríguez-Tzompantzi ${ }^{1, \mathrm{a}}$, Alberto Escalante ${ }^{2, \mathrm{~b}}$ \\ ${ }^{1}$ Facultad de Ciencias Físico-Matemáticas, Benemérita Universidad Autónoma de Puebla, Apartado Postal 1152, 72001 Puebla, PUE, Mexico \\ 2 Instituto de Física, Benemérita Universidad Autónoma de Puebla, Apartado Postal J-48, 72570 Puebla, PUE, Mexico
}

Received: 22 November 2017 / Accepted: 27 April 2018 / Published online: 10 May 2018

(C) The Author(s) 2018

\begin{abstract}
By applying the Faddeev-Jackiw symplectic approach we systematically show that both the local gauge symmetry and the constraint structure of topologically massive gravity with a cosmological constant $\Lambda$, elegantly encoded in the zero-modes of the symplectic matrix, can be identified. Thereafter, via a suitable partial gauge-fixing procedure, the time gauge, we calculate the quantization bracket structure (generalized Faddeev-Jackiw brackets) for the dynamic variables and confirm that the number of physical degrees of freedom is one. This approach provides an alternative to explore the dynamical content of massive gravity models.
\end{abstract}

\section{Introduction}

Fundamental issues in modern cosmology, such as inflation, dark matter, dark energy and the accelerating expansion of our universe [1,2], have long motivated to propose alternative gravity theories beyond original Einstein's General Relativity, both in the ultraviolet (UV) and the infrared (IR) regimes. According to Lovelock's theorem [3,4] any modification of General Relativity requires at least one of the following ingredients: (1) extra dimensions, (2) extra degrees of freedom, (3) higher-derivatives terms, and iv) non-locality. Massive gravity theories are an example of the type-ii ingredients, in which the graviton will acquire a non-zero mass (see e.g. [5-18]). Along these lines, it has long been known that the first massive gravity theory was introduced by Pauli and Fierz [6], where they presented a linear action for a spin-2 field on a four dimensional flat background. Moreover, the Fierz-Pauli theory describes the propagation of a field with five degrees of freedom of positive energy whereas General Relativity theory has two degrees of freedom. However, Boulware and

\footnotetext{
a e-mail: omar.tz2701@gmail.com

be-mail: aescalan@ifuap.buap.mx
}

Deser studied some specific fully non-linear massive gravity theories and pointed out that a general non-linear theory of massive gravity generically contains six propagating degrees of freedom [19]. While the linear theory has five degrees of freedom, the non-linear theories studied by these authors turned out to have an extra degree of freedom, which however is unphysical since it has a negative kinetic energy and renders the whole theory unstable: it was therefore called the Boulware-Deser ghost. After a great effort, a non-linear theory free of such a ghost field was finally obtained by de Rham, Gabadadze and Tolley (dRGT) [7-9]. The advantage of the dRGT model is that it contains two dynamical constraints that eliminate both the ghost field and its canonicallyconjugate momentum. The absence of the Boulware-Deser ghost was shown explicitly by performing the counting of the physical degrees of freedom in the framework of the Hamiltonian formalism [10-12,17,18]. Unfortunately, the Hamiltonian analysis of these models is a difficult task to develop, and its symmetry properties have not been studied yet via a complete analysis of its constraints. On the other hand, in the study of massive gravity models, it is always useful to consider toy models that share the conceptual foundations of the four-dimensional theories, but at the same time are free of technical difficulties; this is particularly true in threedimensional (3D) gravity. In this work, we focus on the simplest 3D version of a massive gravity theory; in this manner, in order to obtain a realistic 3D-Einstein gravity as compared to the higher-dimensional theory, regarding the local propagating modes, one can modify the theory by adding up higher-derivative curvature terms in the Einstein-Hilbert (EH) action, which leads to the simplest 3D-massive gravity theory known as Topologically Massive Gravity (TMG). This theory consists of an EH term, with or without a consmological constant $\Lambda$, plus a parity-violating gravitational ChernSimons (CS) term with coefficient $\frac{1}{\mu}$ [20-25]. At the linear level, without a cosmological constant this theory describes a single massive state of helicity \pm 2 (depending on the rel- 
ative sign between the EH and CS terms) on a Minkowski background $^{1}$ [26], and defines an unitary irreducible representation of the 3D Poincaré group [27].

However, while a linearized analysis usually allows us a reliable counting of the physical degrees of freedom, it can yield misleading results in some cases. A Lagrangian/Hamiltonian formulation should provide a way to count the number of local physical degrees of freedom without resorting to linearization, that is, taking into account all the physical constraints and gauge invariance. In this sense, the identification of the physical degrees of freedom can be addressed by a direct application of Dirac's method for constrained Hamiltonian systems [28], which systematically separates all constraints into first and second-class ones $[29,30]$. As a consequence, the physical degrees of freedom can be separated from the gauge degrees of freedom, and a generator of the gauge symmetry can be constructed out of a combination of first-class constraints [31]. Furthermore, the Dirac brackets to quantize a gauge system can be obtained once the second-class constraints are removed. In the case of the massive gravity theories, however, the separation between first-and second-class constraints is a delicate issue, and the system considered in this paper is not an exception [32-35]. In particular, in Ref. [32] the Hamiltonian structure of TMG was further analyzed via the Dirac formalism. Indeed, these authors obtain the secondary first-class-constraint structure of this model with the help of the Dirac conjecture: "If $\phi$ is a first-class constraint, then $\left\{\phi, H^{T}\right\}$ is also a first-class constraint". Nevertheless, this treatment is quite involved and unsatisfactory. On the other hand, the authors of Refs. [34,35] present a fully Lagrangian analysis, but the right number of physical degrees of freedom in the configuration space can only be obtained once an ad hoc extra constraint on the basic variables is invoked. Hence, it is worth exploring whether all the necessary constraints can be systematically obtained via a Lagrangian formulation, therefore, the analysis of the constraints and the gauge symmetry of massive gravity models, still is missing in the literature.

As an alternative approach to Dirac's method, Faddeev and Jackiw [36] proposed a new framework, which is geometrically well motivated and is based on the symplectic structure for constrained systems. This approach, the so-called Faddeev-Jackiw (F-J) symplectic formalism (for a detailed account see [37-44]), is useful to obtain in an elegant way several essential elements of a particular physical theory, such as the physical constraints, the local gauge symmetry,

\footnotetext{
1 In the presence of a cosmological constant, Minkowski space-time is no longer a vacuum solution and the new maximally symmetric solutions are de Sitter $(\mathrm{dS})$ space-time for positive $\Lambda(\mathrm{dS}$ has $S O(3,1)$ isometry) and anti-de Sitter (AdS) space-time for negative $\Lambda$ (AdS has $S O(2,2)$ isomety). In this respect, the $S O(2,2)$ group can be seen as a $\Lambda$-deformed Poincaré group [56], if $\Lambda \rightarrow 0$ the AdS algebra contracts to the usual Poincaré algebra.
}

the quantization bracket structure and the number of physical degrees of freedom. It turns out that the F-J approach does not require to classify the constraints into first- and secondclass ones as in Dirac's approach is done. Even more, it does not invoke Dirac's conjecture; rather, in this approach, all relevant information can be ontained through a symplectic matrix. For a gauge system, the symplectic matrix remains singular unless a gauge-fixing procedure is introduced. In addition, the generators of the gauge symmetries are given in terms of the zero-modes of the symplectic matrix, thus, the F-J symplectic method provides an effective tool for dealing with gauge theories.

In this manner, the purpose of this article is to present a detailed F-J analysis of three-dimensional topologically massive AdS gravity in a completely different context to that presented in Refs. [32-35]. In particular, we study the nature of the physical constraints and we obtain the gauge symmetry, as well as its generators, under which all the physical quantities must be invariant. Afterwards, we obtain both the fundamental quantization brackets and the number of physical degrees of freedom by introducing an appropriate gaugefixing procedure.

The remainder of this paper is structured as follows. In Sect. 2 we briefly review the topologically massive AdS gravity action. Section 3 is devoted to explore the nature of the constraints within the Faddeev-Jackiw symplectic framework and derive the corresponding symplectic matrix. The full set of physical constraints of the theory are also obtained. In Sect. 4, the gauge symmetry and its generators are obtained via the zero-modes of the symplectic matrix. In Sect. 5 we introduce gauge-fixing conditions in order to obtain both the quantization bracket structure and the number of physical degrees of freedom. In Sect. 6, we conclude with a brief discussion of our results.

\section{Action and equations of motion of topologically massive gravity}

Our starting point is the action of topologically massive AdS gravity written in the first-order formalism:

$$
\begin{aligned}
S[A, e, \lambda]= & \int_{\mathcal{M}}\left[2 \theta e^{i} \wedge F[A]_{i}-\frac{1}{3} \Lambda f_{i j k} e^{i} \wedge e^{j} \wedge e^{k}\right. \\
& \left.+\lambda^{i} \wedge T_{i}+\frac{\theta}{\mu} A^{i} \wedge\left(d A_{i}+\frac{1}{3} f_{i j k} A^{j} \wedge A^{k}\right)\right],
\end{aligned}
$$

where $\mu$ is the Chern-Simons parameter, $\theta=1 / 16 \pi G$ with $G$ the 3D Newton's constant, and $\Lambda$ is a cosmological constant such that $\Lambda=-1 / l^{2}$, where $l$ is the AdS radius [26]. Furthermore, the fundamental fields of this action are: the dreibein 1-form $e^{i}=e_{\mu}^{i} d x^{\mu}$ that determines a space-time 
metric via $g_{\mu \nu}=e_{\mu}{ }^{i} e_{\nu}{ }^{j} \eta_{i j}$; the auxiliary field 1-form $\lambda^{i}$ that ensures that the torsion vanishes $T_{i}=0[45,46]$; and the dualized spin-connection $A^{i}=A_{\mu}{ }^{i} d x^{\mu}$ valued on the adjoint representation of the Lie group $S O(2,2)$, so that, it admits an invariant totally anti-symmetric tensor $f_{i j k}$. The connection acts on internal indices and defines a derivative operator:

$D_{\mu} V^{i} \equiv \partial_{\mu} V^{i}+f^{i}{ }_{j k} A_{\mu}{ }^{j} V^{k}$,

where $\partial$ is a fiducial derivative operator. Finally, $T_{i}$ is the local Lorentz covariant torsion 2-form and $F_{i}$ is the curvature 2 -form of the spin connection $A^{i}$, which explicitly read

$$
T_{i} \equiv d e_{i}+f_{i j k} A^{j} \wedge e^{k}, \quad F_{i} \equiv d A_{i}+\frac{1}{2} f_{i j k} A^{j} \wedge A^{k} .
$$

The convention adopted is the standard one, that is, Greek indices refer to spacetime coordinates and Latin letters correspond to Lorentz indices. The equations of motion that can be extracted by varying the action (1) with respect to $e^{i}, A^{i}$ and $\lambda^{i}$, respectively, in addition to some total derivative terms, are given by

$$
(\delta e)^{\alpha i}=\epsilon^{\alpha \nu \rho}\left(2 \theta F_{\nu \rho}{ }^{i}+D_{\nu} \lambda_{\rho}{ }^{i}-\Lambda f^{i}{ }_{j k} e_{\nu}{ }^{j} e_{\rho}{ }^{k}\right)=0,
$$

$(\delta A)^{\alpha i}=\epsilon^{\alpha \nu \rho}\left(2 \theta T_{\nu \rho}{ }^{i}+f^{i}{ }_{j k} \lambda_{\nu}{ }^{j} e_{\rho}{ }^{k}+2 \theta \mu^{-1} F_{\nu \rho}{ }^{i}\right)=0$,

$$
(\delta \lambda)^{\alpha i}=\epsilon^{\alpha \nu \rho} T_{\nu \rho}^{i}=0 .
$$

One can note that Eq. (6) is the condition for the compatibility of $A_{\mu}{ }^{i}$ and $e_{\mu}{ }^{i}$, which implies

$$
A_{\mu}{ }^{i j}=-e^{v j} \partial_{\mu} e_{\nu}{ }^{i}+\Gamma_{\alpha \mu}^{\beta} e_{\beta}^{i} e^{\alpha j},
$$

with $\Gamma_{\alpha \mu}^{\beta}$ the Christoffel symbols of the metric $g_{\mu \nu}$, and $A_{\mu}{ }^{i j}$ the standard connection obtained by dualizing the $f$-tensor, $A_{\mu}{ }^{i j}=-f^{i j}{ }_{k} A_{\mu}{ }^{k}$. Moreover, by inserting Eq. (6) into Eq. (5), one can solve for the Lagrangian multiplier $\lambda_{\mu}{ }^{i}$ in terms of the 3D Schouten tensor of the manifold $\mathcal{M}$ :

$\lambda_{\mu}{ }^{i}=2 \theta \mu^{-2} S_{\mu \nu} e^{i \nu} \quad$ with $\quad S_{\mu \nu}=R_{\mu \nu}-\frac{1}{4} g_{\mu \nu} R$.

Here, we have made use of the fact that the internal and space-time curvature tensors $F_{\mu \nu}{ }^{i j}$ and $R_{\mu \nu}{ }^{\alpha \beta}$ are related by

$R^{\alpha \beta}{ }_{\mu \nu}=e^{\alpha}{ }_{i} e^{\beta}{ }_{j} F_{\mu \nu}{ }^{i j}$ with $F_{\mu \nu}{ }^{i j}=-f^{i j}{ }_{k} F_{\mu \nu}{ }^{k}$.

After plugging these results into Eq. (4) and a lengthy calculation, one can find the field equation of TMG [20-22] in the second-order formalism:
$G_{\mu \nu}+\frac{1}{\mu} C_{\mu \nu}=0$,

where $G_{\mu \nu}$ is the cosmological-constant-modified Einstein tensor defined as

$G_{\mu \nu} \equiv R_{\mu \nu}-\frac{1}{2} g_{\mu \nu} R+\Lambda g_{\mu \nu}$,

and $C_{\mu \nu}$ is the symmetric traceless Cotton tensor given by

$C_{\mu \nu} \equiv \epsilon_{\mu}{ }^{\alpha \beta} \nabla_{\alpha}\left(R_{\beta \nu}-\frac{1}{4} g_{\beta \nu} R\right)$,

where $\nabla$ is the covariant derivative defined by $\Gamma$. Considering small perturbations around an anti-de Sitter background, this theory describes the presence of a single massive graviton mode [20-22,26,27]. However, from a theoretical point of view, it is better to checkout the validity of such rough arguments by a careful symplectic, Hamiltonian or Lagrangian analysis at nonlinear order.

\section{The nature of the constraints in the Faddeev-Jackiw symplectic framework}

In order to apply the Faddeev-Jackiw's symplectic approach [36], throughout this work we take the spacetime $\mathcal{M}$ to be globally hyperbolic such that it may be foliated as $\mathcal{M} \simeq$ $\Sigma \times \Re$, where $\Sigma$ corresponds to a Cauchy's surface without boundary $(\partial \Sigma=0)$ and $\Re$ represents an evolution parameter. By performing a $2+1$ splitting of our fields without breaking the internal symmetry, the TMG action (1) acquires the form,

$$
\begin{aligned}
S[A, e, \lambda]= & \int\left[\epsilon^{a b} \theta\left(\frac{1}{\mu} A_{b i}+2 e_{b i}\right) \dot{A}^{i}{ }_{a}+\epsilon^{a b} \lambda_{i b} \dot{e}^{i}{ }_{a}\right. \\
& +\epsilon^{a b} e^{i}{ }_{0}\left(\theta F_{a b i}+D_{a} \lambda_{b i}-\Lambda f_{i j k} e_{a}{ }^{j} e_{b}{ }^{k}\right) \\
& +\epsilon^{a b} A^{i}{ }_{0}\left(\theta T_{a b i}+\frac{1}{\mu} \theta F_{a b i}+f_{i j k} \lambda^{j}{ }_{a} e^{k}{ }_{b}\right) \\
& \left.+\frac{1}{2} \epsilon^{a b} \lambda^{i}{ }_{0} T_{a b i}\right] d^{3} x,
\end{aligned}
$$

up to a boundary term. Here $F_{a b}{ }^{i}=\partial_{a} A_{b}^{i}-\partial_{b} A_{a}^{i}+$ $f^{i}{ }_{j k} A_{a}{ }^{j} A_{b}{ }^{k}$ is the field strength of $A_{a}{ }^{i}, T_{a b}{ }^{i}=D_{a} e_{b}{ }^{i}-$ $D_{b} e_{a}{ }^{i}$ and $D_{a} \lambda_{b}{ }^{i}=\partial_{a} \lambda_{b}{ }^{i}+f^{i}{ }_{i j} A_{a}{ }^{j} \lambda_{b}{ }^{k}$. Besides $a, b, c, \ldots$ are space coordinates and the dot denotes a derivative with respect to the evolution parameter. We can read off the Lagrangian density from (13) as

$$
\begin{aligned}
\mathcal{L}^{(0)}= & \epsilon^{a b} \theta\left(\frac{1}{\mu} A_{b i}+2 e_{b i}\right) \dot{A}^{i}{ }_{a}+\epsilon^{a b} \lambda_{i b} \dot{e}^{i}{ }_{a} \\
& +\epsilon^{a b} e^{i}{ }_{0}\left(\theta F_{a b i}+D_{a} \lambda_{b i}-\Lambda f_{i j k} e_{a}{ }^{j} e_{b}{ }^{k}\right)+\frac{1}{2} \epsilon^{a b} \lambda^{i}{ }_{0} T_{a b i} \\
& +\epsilon^{a b} A^{i}{ }_{0}\left(\theta T_{a b i}+\frac{1}{\mu} \theta F_{a b i}+f_{i j k} \lambda^{j}{ }_{a} e^{k}{ }_{b}\right) .
\end{aligned}
$$


In particular, this Lagrangian density can be expressed in a compact form

$\mathcal{L}^{(0)}=a_{I}^{(0)} \dot{\xi}^{(0) I}-V^{(0)}$,

where an initial set of symplectic variable is introduced as follows

$\xi^{(0) I}=\left(A_{a}^{i}, A_{0}^{i}, e_{a}^{i}, e_{0}^{i}, \lambda_{a}^{i}, \lambda_{0}^{i}\right)$

which allows us to identify the corresponding symplectic one-form

$a_{I}^{(0)}=\left(\epsilon^{a b} \theta\left(\frac{1}{\mu} A_{b i}+2 e_{b i}\right), 0, \epsilon^{a b} \lambda_{b i}, 0,0,0\right)$,

whereas the symplectic potential reads

$$
\begin{aligned}
V^{(0)}= & -\epsilon^{a b} e^{i}{ }_{0}\left(\theta F_{a b i}+D_{a} \lambda_{b i}-\Lambda f_{i j k} e_{a}{ }^{j} e_{b}{ }^{k}\right) \\
& -\frac{1}{2} \epsilon^{a b} \lambda^{i}{ }_{0} T_{a b i}-\epsilon^{a b} A^{i}{ }_{0} \\
& \times\left(\theta T_{a b i}+\frac{1}{\mu} \theta F_{a b i}+f_{i j k} \lambda^{j}{ }_{a} e^{k}{ }_{b}\right) .
\end{aligned}
$$

On the other hand, the corresponding equations of motion arising from the above Lagrangian (15) can be written as

$f_{I J}^{(0)} \dot{\xi}^{(0) J}-\frac{\delta}{\delta \xi^{(0) I}} V(\xi)^{(0)}=0$,

with $f_{I J}^{(0)} \equiv \frac{\delta}{\delta \xi^{(0) I}} a_{J}^{(0)}-\frac{\delta}{\delta \xi^{(0) J}} a_{I}^{(0)}$ the two-form symplectic matrix associated with $\mathcal{L}^{(0)}$, which is clearly antisymmetric (see the appendix). By using the symplectic variables (16) and (17), we find that the corresponding symplectic matrix $f_{I J}^{(0)}(x, y)$ can be written as

$$
\left(\begin{array}{llllll}
2 \frac{\theta}{\mu} \epsilon^{a b} \eta_{i j} & 0 & -2 \theta \epsilon^{a b} \eta_{i j} & 0 & 0 & 0 \\
0 & 0 & 0 & 0 & 0 & 0 \\
2 \theta \epsilon^{a b} \eta_{i j} & 0 & 0 & 0 & -\epsilon^{a b} \eta_{i j} & 0 \\
0 & 0 & 0 & 0 & 0 & 0 \\
0 & 0 & \epsilon^{a b} \eta_{i j} & 0 & 0 & 0 \\
0 & 0 & 0 & 0 & 0 & 0
\end{array}\right) \delta^{2}(x-y) .
$$

It is not difficult to see that the matrix $f_{I J}^{(0)}$ is degenerate in the sense that there are more degrees of freedom in the equations of motion (19) than physical degrees of freedom in the theory. In this case, there are constraints that must remove the unphysical degrees of freedom. In this formalism the constraints emerge as algebraic relations necessary to maintain the consistency of the equations of motion. Moreover, it is straightforward to determine that the zero-modes of the singular matrix (20) are $\left(v_{1}^{(0)}\right)^{I}=\left(0, v^{A^{i}}, 0,0,0,0\right),\left(v_{2}^{(0)}\right)^{I}=\left(0,0,0, v^{e^{i}}, 0,0\right)$ and $\left(v_{3}^{(0)}\right)^{I}=\left(0,0,0,0,0, v^{\lambda^{i} 0}\right)$, with non-vanishing arbitrary components $v^{A^{i} 0}, v^{e^{i}}$ and $v^{\lambda^{i} 0}$, respectively.

The zero-modes satisfy the equation $\left(v_{1,2,3}^{(0)}\right)^{I} f_{I J}^{(0)}=0$, and from the equation of motion (19), we have the following constraint relations:

$$
\begin{aligned}
& \int d x^{2}\left(v_{1}^{(0)}\right)_{J}^{T} \frac{\delta}{\delta \xi^{J}} \int d y^{2} V^{(0)} \\
& =v^{A_{0}^{i}}\left(\theta \epsilon^{a b} T_{a b i}+\frac{\theta}{\mu} \epsilon^{a b} F_{a b i}+\epsilon^{a b} f_{i j k} \lambda^{j}{ }_{a} e^{k}{ }_{b}\right)=0 \\
& \int d x^{2}\left(v_{2}^{(0)}\right)_{J}^{T} \frac{\delta}{\delta \xi^{J}} \int d y^{2} V^{(0)} \\
& =v^{e^{i}}\left(\theta \epsilon^{a b} F_{a b i}+\epsilon^{a b} D_{a} \lambda_{b i}-\Lambda \epsilon^{a b} f_{i j k} e_{a}{ }^{j} e_{b}{ }^{k}\right)=0 \\
& \int d x^{2}\left(v_{3}^{(0)}\right)_{J}^{T} \frac{\delta}{\delta \xi^{J}} \int d y^{2} V^{(0)}=v^{\lambda_{0}^{i}}\left(\frac{1}{2} \epsilon^{a b} T_{a b i}\right)=0,
\end{aligned}
$$

where $v^{A_{0}^{i}}, v^{e_{0}^{i}}$ and $v^{\lambda_{0}^{i}}$ are arbitrary functions. The constraints become

$\Xi_{i}^{(0)}=\theta \epsilon^{a b} T_{a b i}+\frac{\theta}{\mu} \epsilon^{a b} F_{a b i}+\epsilon^{a b} f_{i j k} \lambda^{j}{ }_{a} e^{k}{ }_{b}=0$,

$\Theta_{i}^{(0)}=\theta \epsilon^{a b} F_{a b i}+\epsilon^{a b} D_{a} \lambda_{b i}-\Lambda \epsilon^{a b} f_{i j k} e_{a}^{j} e_{b}{ }^{k}=0$,

$\Sigma_{i}^{(0)}=\frac{1}{2} \epsilon^{a b} T_{a b i}=0$.

Now, according to the methodology of the symplectic framework, we will analyze whether there are new constraints. Thus, we demand stability (consistency condition) of the constraints (A8), (25) and (26), which guarantees their timeindependence. Since $\Xi_{i}, \Theta_{i}$ and $\Sigma_{i}$ depend only on the set of symplectic variables $\xi^{(0) I}$, the consistency condition can be written as

$$
\dot{\Omega}^{(0)}=\frac{\delta \Omega^{(0)}}{\delta \xi^{(0) I}} \dot{\xi}^{(0) I}=0, \quad \text { with } \Omega^{(0)}=\Xi_{i}^{(0)}, \Theta_{i}^{(0)}, \Sigma_{i}^{(0)} .
$$

Therefore the consistency of the constraints $\Omega^{(0)}$, together with the equations of motion (19) can be generally rewritten as

$f_{K J}^{(1)} \dot{\xi}^{(0) J}=Z_{K}^{(1)}(\xi)$,

with

$f_{K J}^{(1)}=\left(\begin{array}{c}f_{I J}^{(0)} \\ \frac{\delta}{\delta \xi^{(0) I}} \Omega^{(0)}\end{array}\right) \quad$ and $\quad Z_{K}^{(1)}=\left(\begin{array}{c}\frac{\delta}{\delta \xi^{(0) I}} V^{(0)} \\ 0 \\ 0 \\ 0\end{array}\right)$. 


$$
\begin{gathered}
\left(\begin{array}{cccccc}
2 \frac{\theta}{\mu} \eta_{i j} & 0 & -2 \theta \eta_{i j} & 0 & 0 & 0 \\
0 & 0 & 0 & 0 & 0 & 0 \\
2 \theta \eta_{i j} & 0 & 0 & 0 & -\eta_{i j} & 0 \\
0 & 0 & 0 & 0 & 0 & 0 \\
0 & 0 & \eta_{i j} & 0 & 0 & 0 \\
0 & 0 & 0 & 0 & 0 & 0 \\
2 \frac{\theta}{\mu}\left(\eta_{i j} \partial_{a}-f_{i j k} A^{k}{ }_{a}-\mu f_{i j k} e^{k}{ }_{a}\right) & 0 & 2 \theta\left(\eta_{i j} \partial_{a}-f_{i j k} A^{k}{ }_{a}-\frac{1}{2 \theta} f_{i j k} \lambda^{k}{ }_{a}\right) & 0 & -f_{i j k} e_{a} & 0 \\
2 \theta\left(\eta_{i j} \partial_{a}-f_{i j k} A_{a}^{k}-\frac{1}{2 \theta} f_{i j k} \lambda^{k}{ }_{a}\right) & 0 & 2 \Lambda f_{i j k} e_{a}{ }^{k} & 0 & \left(\eta_{i j} \partial_{a}-f_{i j k} A_{a}^{k}\right) & 0 \\
-f_{i j k} e^{k}{ }_{a} & 0 & \left(\eta_{i j} \partial_{a}-f_{i j k} A_{a}^{k}\right) & 0 & 0 & 0
\end{array}\right) \\
\times \epsilon^{a b} \delta^{2}(x-y) .
\end{gathered}
$$

It is clear that $f_{K J}^{(1)}$ is not a square matrix, however, it has linearly independent zero-modes, which turn out to be

$$
\begin{aligned}
\left(v_{1}^{(1)}\right)^{K}= & \left(-\partial_{a} \eta_{m}^{j}-f^{j}{ }_{l m} A_{a}^{l}, 0,-f^{j}{ }_{l m} e_{a}^{l}, 0,\right. \\
& \left.-f^{j}{ }_{l m} \lambda_{a}{ }^{l}, 0, \eta_{m}^{j}, 0,0\right), \\
\left(v_{2}^{(1)}\right)^{K}= & \left(-\frac{\mu}{2 \theta} f^{j}{ }_{l m} \lambda_{a}^{l}, 0,-\partial_{a} \eta_{m}^{j}-f^{j}{ }_{l m} A_{a}^{l},\right. \\
& \left.0, f^{j}{ }_{l m}\left(\mu \lambda^{l}{ }_{a}+2 \Lambda e^{l}{ }_{a}\right), 0,0, \eta_{m}^{j}, 0\right), \\
\left(v_{3}^{(1)}\right)^{K}= & \left(-\frac{\mu}{2 \theta} f^{j}{ }_{{ }_{l m}} e_{a}^{l}, 0,0,0,-\partial_{a} \eta_{m}^{j}\right. \\
& \left.-f^{j}{ }_{l m} A^{l}{ }_{a}+\mu f^{j}{ }_{l m} e_{a}^{l}, 0,0,0, \eta_{m}^{j}\right),
\end{aligned}
$$

such that $\left(v_{1,2,3}^{(1)}\right)^{K} f_{K J}^{(1)}=0$. By using the symplectic potential, we find that the matrix $Z_{K}^{(1)}$ is given by
The substitution $\Omega^{(0)}=0$ guarantees that these constraints will drop from the remainder of the calculation. Then, from (36) and (37), together with the invertibility of $e_{\alpha i}$ and $\lambda_{\alpha i}$, we finally obtain

$\Phi^{\alpha}=\epsilon^{\alpha \beta \gamma} e_{\beta}{ }^{j} \lambda_{\gamma j}=0$,

which are known as symmetry conditions and play a crucial role in the relation of the metric and tetrad formulations of massive gravity theories and multi-bigravity ones [13]. Furthermore, one finds that the Eq. (38) can be split into two equations:

$\Phi^{a}=\epsilon^{a b}\left(e_{0}^{i} \lambda_{i b}-e^{i}{ }_{b} \lambda_{i 0}\right)=0$,

$$
\left(\begin{array}{c}
-2 \theta\left(D_{a} e_{0 j}+\frac{1}{\mu} D_{a} A_{0 j}\right)+f_{j l m}\left(e_{0}{ }^{l} \lambda_{a}{ }^{m}+\left(\lambda_{0}{ }^{l}+2 \theta A_{0}{ }^{l}\right) e_{a}{ }^{m}\right) \\
\Xi_{i}^{(0)} \\
-D_{a} \lambda_{0 j}-2 \theta D_{a} A_{0 j}+f_{j l m} A_{0} \lambda^{m}{ }_{a}-2 \Lambda f_{j l m} e^{l} e_{0}{ }_{a} \\
\Theta_{i}^{(0)} \\
-D_{a} e_{0 j}+f_{j l m} A^{l} e_{0} e_{a} \\
\Sigma_{i}^{(0)} \\
0 \\
0 \\
0
\end{array}\right) \epsilon^{a b} \delta^{2}(x-y)
$$

By multiplying both sides of Eq. (28) by the zero-modes of the matrix $f_{K J}^{(1)}$, and evaluating at $\Omega^{(0)}=0$, we get the following covariant constraint relations (the integration symbol $\int$ is omitted for clarity):

$$
\begin{aligned}
& \left.\left(v_{1}^{(1)}\right)^{K} Z_{K}^{(1)}\right|_{\Omega^{(0)}=0}=0, \\
& \left.\left(v_{2}^{(1)}\right)^{K} Z_{K}^{(1)}\right|_{\Omega^{(0)}=0}=-\frac{1}{2 \theta} \mu \epsilon^{\alpha \beta \gamma} \lambda_{\alpha i} e_{\beta}{ }^{j} \lambda_{\gamma j}, \\
& \left.\left(v_{3}^{(1)}\right)^{K} Z_{K}^{(1)}\right|_{\Omega^{(0)}=0}=\frac{1}{2 \theta} \mu \epsilon^{\alpha \beta \gamma} e_{\alpha i} e_{\beta}{ }^{j} \lambda_{\gamma j} .
\end{aligned}
$$

$\Phi^{0}=\epsilon^{a b} e_{a}^{i} \lambda_{i b}=0$.

We can see that the Eq. (39) has fixed fields $e_{0}^{i}$ and $\lambda_{0}^{i}$, whereas Eq. (40) gives us one more constraint. This agrees completely with what was found in [32] by means of the Dirac procedure, however, in that formalism the constraints (39) and (40) arise as tertiary constraints, whereas in $[34,35]$ the constraint (40) was introduced by hand. Now, by imposing the stability 
condition on the new constraint (40), we have the following equation:

$f_{K J}^{(2)} \dot{\xi}^{(0) J}=Z_{K}^{(2)}(\xi)$,

where the matrices $f_{K J}^{(2)}$ and $Z_{K}^{(1)}$ can be expressed as

$f_{K J}^{(2)}=\left(\begin{array}{c}f_{I J}^{(1)} \\ \frac{\delta}{\delta \xi^{(0) I}} \Phi^{0}\end{array}\right) \quad$ and $\quad Z_{K}^{(2)}=\left(\begin{array}{c}Z_{K}^{(1)} \\ 0\end{array}\right)$.

Consequently, the new matrix $f_{I J}^{(2)}$ is given by the following scalar constraint:

$\Upsilon=3 \Lambda+\mu \lambda=0$

which is also in agreement with what was obtained in Ref. [32] via the Dirac procedure, whereas in $[34,35]$ such a constraint is missing. Once again, we can introduce the consistency condition on (49) and explore whether there are further constraints in the theory. Thus, we study the equation

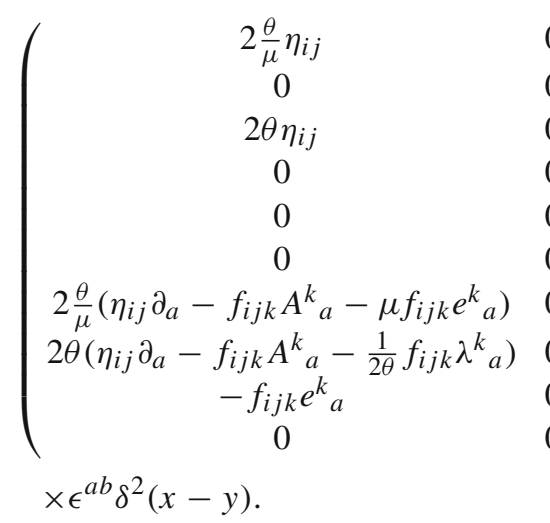

$\times \epsilon^{a b} \delta^{2}(x-y)$.

$\begin{array}{cccc}$\cline { 2 - 3 }$\left.-2 \theta \eta_{i j} & 0 & 0 & 0 \\ 0 & 0 & 0 & 0 \\ 0 & 0 & -\eta_{i j} & 0 \\ 0 & 0 & 0 & 0 \\ \eta_{i j} & 0 & 0 & 0 \\ 0 & 0 & 0 & 0 \\ \left.f_{i j k} A_{a}^{k}-\frac{1}{2 \theta} f_{i j k} \lambda^{k}{ }_{a}\right) & 0 & -f_{i j k} e_{a}^{k} & 0 \\ 2 \Lambda f_{i j k} e_{a}{ }^{k} & 0 & \left(\eta_{i j} \partial_{a}-f_{i j k} A_{a}^{k}\right) & 0 \\ \left.\partial_{a}-f_{i j k} A_{a}\right) & 0 & 0 & 0 \\ \lambda_{a j} & 0 & e_{a j} & 0\end{array}\right)$

One can easily verify that $f_{I J}^{(2)}$ is also a singular matrix that has the following linearly independent zero-modes:

$$
\begin{aligned}
\left(v_{1}^{(2)}\right)^{J}= & \left(-\partial_{a} \eta_{m}^{j}-f^{j}{ }_{l m} A_{a}^{l}, 0,-f^{j}{ }_{l m} e_{a}^{l}, 0\right. \\
& \left.-f^{j}{ }_{l m} \lambda_{a}{ }^{l}, 0, \eta_{m}^{j}, 0,0,0\right), \\
\left(v_{2}^{(2)}\right)^{J}= & \left(-\frac{\mu}{2 \theta} f^{j}{ }_{l m} \lambda^{l}{ }_{a}, 0,-\partial_{a} \eta_{m}^{j}-f^{j}{ }_{l m} A^{l}{ }_{a}, 0, f^{j}{ }_{l m}\right. \\
& \left.\times\left(\mu \lambda^{l}{ }_{a}+2 \Lambda e^{l}{ }_{a}\right), 0,0, \eta_{m}^{j}, 0,0\right), \\
\left(v_{3}^{(2)}\right)^{J}= & \left(-\frac{\mu}{2 \theta} f^{j}{ }_{l m} e_{a}^{l}, 0,0,0,-\partial_{a} \eta_{m}^{j}-f^{j}{ }_{l m} A_{a}^{l}\right. \\
& \left.+\mu f^{j}{ }_{l m} e^{l}{ }_{a}, 0,0,0, \eta_{m}^{j}, 0\right), \\
\left(v_{4}^{(2)}\right)^{J}= & \left(\mu e_{a}{ }^{j}, 0, e_{a}{ }^{j}, 0,\right. \\
& \left.-\left(\lambda_{a}{ }^{j}+2 \theta \mu e_{a}{ }^{j}\right), 0,0,0,0, \eta_{m}^{j}\right) .
\end{aligned}
$$

After performing the contraction of (41) with the zero-modes (44), (45) and (46), it is not difficult to see that these modes do not generate any new constraints, whereas from the zeromode $\left(v_{4}^{(2)}\right)^{J}$ we have the following constraint relation:

$$
\begin{aligned}
\left.\left(v_{4}^{(2)}\right)^{K} Z_{K}^{(2)}\right|_{\Omega^{(0)}, \Omega^{(1)}=0} & =\epsilon^{\alpha \beta v} f_{i j k} e_{\alpha}^{i} e_{\beta}^{j}\left(\Lambda e_{v}^{k}+\mu \lambda_{v}^{k}\right) \\
& =-2 \mathbf{e}(3 \Lambda+\mu \lambda)=0
\end{aligned}
$$

where we have used $\epsilon^{\alpha \beta v} e_{\alpha}{ }^{i} e_{\beta}{ }^{j} e_{\nu}{ }^{k}=\mathbf{e} f^{i j k}$ with $\mathbf{e}=\operatorname{det} \mid$ $e_{\alpha}{ }^{i} \mid$ and $\lambda=e_{\alpha}{ }^{i} \lambda^{\alpha}{ }_{i}$. Hence, from Eq. (48) we can identify
$f_{K J}^{(3)} \dot{\xi}^{(0) J}=Z_{K}^{(3)}(\xi)$

It is easy to verify that even after inserting the above constraint into the matrix $f_{K J}^{(3)}$ and calculating its zero-modes, no new constraint is obtained. Hence, there are no further constraints in the theory and thus our procedure to obtain new constraints via the consistency condition has finished. With the above results and the F-J method, we can now introduce the constraints (A8), (25), (26), (40) and (49) into the Lagrangian density (14) by means of the corresponding Lagrangian multipliers in order to construct a new one. Hence, the new symplectic Lagrangian can be written as

$$
\begin{aligned}
\mathcal{L}= & \epsilon^{a b} \theta\left(\frac{1}{\mu} A_{b i}+2 e_{b i}\right) \dot{A}_{a}^{i}+\epsilon^{a b} \lambda_{i b} \dot{e}_{a}^{i}-\Xi_{i} \dot{\beta}^{i} \\
& -\Theta_{i} \dot{\alpha}^{i}-\Sigma_{i} \dot{\gamma}^{i}-\Phi^{0} \dot{\varphi}_{0}-\Upsilon \dot{\varphi}-V,
\end{aligned}
$$

with $\dot{\alpha}^{i}, \dot{\beta}^{i}, \dot{\gamma}^{i}, \dot{\varphi}_{0}$ and $\dot{\varphi}$ the Lagrangian multipliers relative to the resulting constraints. Furthermore, one can note that the symplectic potential vanishes on the constraint surface since it turns out to be a linear combination of constraints reflecting the general covariance of the theory, that is, $V=$ $\left.V^{(0)}\right|_{\Omega^{(0)}, \Omega^{(1)}, \Phi^{0}, \Upsilon=0}=0$. Moreover, from the Lagrangian density (51), the new symplectic variable set is taken as

$\xi^{I}=\left(A_{a}^{i}, \beta^{i}, e_{a}^{i}, \alpha^{i}, \lambda_{a}^{i}, \gamma^{i}, \varphi^{0}, \varphi\right)$ 
whose corresponding canonical 1 -form is given by

$$
\begin{aligned}
& a_{I}=\left(\epsilon^{a b} \theta\left(\frac{1}{\mu} A_{b i}+2 e_{b i}\right),\right. \\
& \left.-\Xi_{i}, \epsilon^{a b} \lambda_{b i},-\Theta_{i}, 0,-\Sigma_{i},-\Phi_{0},-\Upsilon\right) .
\end{aligned}
$$

We can then use the symplectic variables (52) and (53) to construct the corresponding square symplectic matrix $f_{I J} \equiv$ $\frac{\delta}{\delta \xi^{I}} a_{J}-\frac{\delta}{\delta \xi^{J}} a_{I}$, which turns out to be
$\delta_{G} \xi^{I}=\left(v_{A}\right)^{I} \epsilon^{A}$

where $\left(v_{A}\right)$ are the independent zero-modes of the singular symplectic matrix $f_{I J}$ and $\epsilon^{A}$ are the gauge parameters. For the singular symplectic matrix (54), these zero-modes turn out to be

$$
\left(\begin{array}{cccccccc}
2 \frac{\theta}{\mu} \eta_{i j} & -2 \frac{\theta}{\mu} \nabla_{a i j} & -2 \theta \eta_{i j} & -2 \theta \triangle_{a i j} & 0 & E_{a i j} & 0 & 0 \\
2 \frac{\theta}{\mu} \nabla_{a i j} & 0 & 2 \theta \triangle_{a i j} & 0 & -E_{a i j} & 0 & 0 & 0 \\
2 \theta \eta_{i j} & -2 \theta \triangle_{a i j} & 0 & -2 \Lambda E_{a i j} & -\eta_{i j} & -D_{a i j}^{x} & -\lambda_{a j} & -\mu \epsilon_{a d} \lambda^{d}{ }_{j} \\
2 \theta \triangle_{a i j} & 0 & 2 \Lambda E_{a i j} & 0 & D_{a i j}^{y} & 0 & 0 & 0 \\
0 & E_{a i j} & \eta_{i j} & -D_{a i j}^{x} & 0 & 0 & -e_{a j} & -\mu \epsilon_{a d} e^{d}{ }_{j} \\
-E_{a i j} & 0 & D_{a i j}^{y} & 0 & 0 & 0 & 0 & 0 \\
0 & 0 & \lambda_{a j} & 0 & e_{a j} & 0 & 0 & 0 \\
0 & 0 & \mu \epsilon_{a d} \lambda^{d}{ }_{j} & 0 & \mu \epsilon_{a d} e^{d}{ }_{j} & 0 & 0 & 0
\end{array}\right) \epsilon^{a b} \delta^{2}(x-y)
$$

Here, we have defined $\nabla_{a i j}=\left(D_{a i j}-\mu E_{a i j}\right)$ and $\triangle_{a i j}=$ $\left(D_{a i j}-\frac{1}{2 \theta} L_{a i j}\right)$ with $D_{a i j}=\partial_{a} \eta_{i j}-f_{i j k} A_{a}^{k}, E_{a i j}=$ $f_{i j k} e^{k}{ }_{a}$ and $L_{a i j}=f_{i j k} \lambda^{k}{ }_{a}$ respectively. It is worth noting that the symplectic matrix $f_{I J}$ remains singular, and therefore it still has linearly independent zero-modes. Nevertheless, we have shown that no more constraints are obtained via the consistency conditions. The non-invertibility of $f_{I J}$ is then due to a gauge symmetry that must be fixed via additional conditions (gauge conditions) in order to remove the singularity. In this way the quantization-bracket structure can be determined and the procedure can be achieved in terms of the physical degrees of freedom.

\section{Gauge transformations}

It is well-know that the concept of gauge symmetry has played a central role in the development of fundamental theories of physical laws. On the other hand, the need to describe the interactions through relativistic dynamics led us to build a covariant language with a gauge symmetry [47]. We thus proceed towards the discussion of the gauge symmetry in the symplectic framework. It is worth noting that, when all the constraints have been considered and the symplectic matrix still has zero-modes but no new constraint are obtained, one is led to conclude that the theory must have a local gauge symmetry, therefore, the zero-modes act as the generators of the corresponding gauge symmetry ' $\delta_{G}$ ', that is, the components of the zero-modes provide the transformation rules related to the underlying (gauge) symmetry [38-40]. The local infinitesimal transformations of the symplectic variables generated by $(v)^{I}$ can be expressed as

$$
\begin{aligned}
\left(v_{1}\right)^{I}= & \left(-\partial_{a} \eta_{k}^{j}-f^{j}{ }_{l k} A_{a}{ }^{l}, \eta^{j}{ }_{k},-f^{j}{ }_{l k} e_{a}^{l}, 0\right. \\
& \left.-f^{j}{ }_{l k} \lambda_{a}{ }^{l}, 0,0,0\right) \\
\left(v_{2}\right)^{I}= & \left(-\frac{\mu}{2 \theta} f^{j}{ }_{l k} \lambda_{a}{ }^{l}, 0,-\partial_{a} \eta_{k}^{j}-f^{j}{ }_{l k} A_{a}{ }^{l}, \eta^{j}{ }_{k}, f^{j}{ }_{l k}\right. \\
& \left.\times\left(\mu \lambda_{a}{ }^{l}+2 \Lambda e_{a}{ }^{l}\right), 0,0,0\right) \\
\left(v_{3}\right)^{I}= & \left(-\frac{\mu}{2 \theta} f^{j}{ }_{l k} e_{a}^{l}, 0,0,0,-\partial_{a} \eta_{k}^{j}-f^{j}{ }_{l k} A_{a}{ }^{l}\right. \\
& \left.+\mu f^{j}{ }_{l k} e_{a}{ }^{l}, \eta^{j}{ }_{k}, 0,0\right)
\end{aligned}
$$

which are orthogonal to the gradient of the symplectic potential and at the same time generate local displacements on the isopotential surface. As one can infer from (55), the infinitesimal gauge transformations of the theory are given by

$$
\begin{aligned}
\delta_{G} A_{\alpha}{ }^{i}(x)= & -D_{\alpha} \zeta^{i}-\frac{\mu}{2 \theta} f^{i}{ }_{j k}\left(e_{\alpha}{ }^{j} \varsigma^{k}+\lambda_{\alpha}{ }^{j} \kappa^{k}\right) \\
\delta_{G} e_{\alpha}{ }^{i}(x)= & -D_{\alpha} \kappa^{i}-f^{i}{ }_{j k} e_{a}^{j} \zeta^{k} \\
\delta_{G} \lambda_{\alpha}{ }^{i}(x)= & -D_{\alpha} \varsigma^{i}-f^{i}{ }_{j k} \lambda_{\alpha}{ }^{j} \zeta^{k}+\mu f^{i}{ }_{j k} \\
& \times\left(\lambda_{a}{ }^{j} \kappa^{k}+e_{a}{ }^{j} \zeta^{k}\right)+2 \Lambda f^{i}{ }_{j k} e_{a}{ }^{j} \kappa^{k}
\end{aligned}
$$

where $\zeta^{i}, \kappa^{i}$ and $\zeta^{i}$ are the time-dependent gauge parameters. It is worth remarking that (59), (60) and (61) determine the fundamental gauge symmetries of the theory, which correspond to $\lambda$-deformed $S O(2,2)$ transformations [32]. In these transformations the diffeomorphisms have not been found explicitly. However, it is well known that an appropriate choice of the gauge parameters does generate the diffeomorphism (on-shell) $[47,48,50]$. Let us redefine the gauge parameters as

$$
\zeta^{i}=-A^{i}{ }_{\mu} \varepsilon^{\mu}, \quad \kappa^{i}=-e_{\mu}^{i} \varepsilon^{\mu}, \quad \zeta^{i}=-\lambda^{i}{ }_{\mu} \varepsilon^{\mu},
$$


with $\varepsilon^{\mu}$ an arbitrary three-vector. Hence, from the fundamental gauge symmetry (61) and the mapping (62), we obtain

$$
\begin{aligned}
\delta_{G} A_{\alpha}{ }^{i}= & \mathfrak{L}_{\varepsilon} A_{\alpha}{ }^{i}+\mu \varepsilon^{\mu} \epsilon_{\alpha \mu \nu}\left[\frac{1}{2 \theta}(\delta A)^{v i}+(\delta \lambda)^{v i}\right], \\
\delta_{G} e_{\alpha}{ }^{i}= & \mathfrak{L}_{\varepsilon} e_{\alpha}{ }^{i}-\varepsilon^{\mu} \epsilon_{\alpha \mu \nu}(\delta \lambda)^{v i}, \\
\delta_{G} \lambda_{\alpha}{ }^{i}= & \mathfrak{L}_{\varepsilon} \lambda_{\alpha}{ }^{i}+2 \mu \theta \varepsilon^{\mu} \epsilon_{\alpha \mu \nu} \\
& \times\left[\frac{1}{2 \mu \theta}(\delta e)^{v i}-\frac{1}{2 \theta}(\delta A)^{v i}+(\delta \lambda)^{v i}\right] .
\end{aligned}
$$

which correspond precisely (on-shell) to diffeomorphisms. In addition, TMG (1) is also made invariant under Poincaré transformations by construction $[47,48]$. Thus, in order to recover the Poincaré symmetry, we need to map the arbitrary gauge parameters of the fundamental gauge symmetry ' $\delta_{G}$ ', (61) into those of the Poincaré symmetry. This is achieved by a mapping of the gauge parameters [48-50], e.g.:

$\zeta^{i}=A_{\mu}^{i} \varepsilon^{\mu}+\omega^{i}, \quad \kappa^{i}=e^{i}{ }_{\mu} \varepsilon^{\mu}, \quad \zeta^{i}=\lambda^{i}{ }_{\mu} \varepsilon^{\mu}$

such that $\varepsilon^{\mu}$ and $\omega^{i}$ are related to local coordinate translations and local Lorentz rotations, respectively, all together constitute the 6 independent gauge parameters of Poincaré symmetries in 3D. By using this map, the gauge symmetries reproduce the Poincaré symmetries modulo terms proportional to the equations of motion

$$
\begin{aligned}
\delta_{G} e_{\alpha}{ }^{i}= & -\varepsilon^{\mu} \partial_{\mu} e_{\alpha}{ }^{i}-e_{\mu}{ }^{i} \partial_{\alpha} \varepsilon^{\mu}-f^{i}{ }_{j k} e_{\alpha}{ }^{j} \omega^{k} \\
& +\varepsilon^{\gamma} \epsilon_{\alpha \gamma \nu}(\delta \lambda)^{\nu i}, \\
\delta_{G} A_{\alpha}{ }^{i}= & -\partial_{\alpha} \omega^{i}-f^{i}{ }_{j k} A_{\alpha}{ }^{j} \omega^{k}-\varepsilon^{\mu} \partial_{\mu} A_{\alpha}{ }^{i}-A_{\mu}{ }^{i} \partial_{\alpha} \varepsilon^{\mu} \\
& -\mu \varepsilon^{\gamma} \epsilon_{\alpha \gamma \nu}\left[\frac{1}{2 \theta}(\delta A)^{v i}+(\delta \lambda)^{\nu i}\right], \\
\delta_{G} \lambda_{\alpha}{ }^{i}= & -\varepsilon^{\mu} \partial_{\mu} \lambda_{\alpha}{ }^{i}-\lambda_{\mu}{ }^{i} \partial_{\alpha} \varepsilon^{\mu}-f^{i}{ }_{j k} \lambda_{\alpha}{ }^{j} \omega^{k}-2 \mu \theta \varepsilon^{\gamma} \epsilon_{\alpha \gamma \nu} \\
& \times\left[\frac{1}{2 \mu \theta}(\delta e)^{v i}-\frac{1}{2 \theta}(\delta A)^{v i}+(\delta \lambda)^{v i}\right],
\end{aligned}
$$

where the equations of motion $(\delta e)^{v i},(\delta A)^{v i}$ and $(\delta \lambda)^{v i}$ are defined in (4)-(6). We thus conclude that the Poincaré symmetry (65) as well as the diffeomorphisms (62) are not independent symmetries: they are contained indeed in the fundamental gauge symmetry (61) as on-shell symmetries, that is, only when the equations of motion are imposed. In addition, the generators of such gauge transformations can be represented in terms of the zero-modes, thereby making evident that the zero-modes of the symplectic two-form encode all the information about the gauge structure of this theory. It is important to comment that the gauge symmetry (65) was reported in [32] by means of a different way.

\section{The Faddeev-Jackiw brackets and degrees of freedom counting}

As was already mentioned in Sect. 3, if a gauge symmetry is present then the symplectic matrix obtained at the end of the procedure is still singular. Nevertheless, in order to obtain a non-singular symplectic matrix and to determine the quantization bracket (F-J brackets) structure between the dynamical fields, we must impose a gauge-fixing procedure, that is, new gauge constraints. In this case, we now partially fix the gauge by imposing the time-gauge, namely, $A_{0}^{i_{0}}=0$, $e^{i}{ }_{0}=0, \lambda^{i}{ }_{0}=0$ and $\varphi_{0}=$ cte (i.e. $\left.\dot{\varphi}_{0}=0\right)$. In this manner, we also introduce new Lagrange multipliers that enforce these gauge conditions, namely, $\dot{\rho}_{i}, \dot{\omega}_{i}, \dot{\tau}_{i}$ and $\dot{\sigma}^{0}$. Thus, the final symplectic Lagrangian after gauge fixing can be written as

$$
\begin{aligned}
\mathcal{L}= & \epsilon^{a b} \theta\left(\frac{1}{\mu} A_{b i}+2 e_{b i}\right) \dot{A}_{a}^{i}+\epsilon^{a b} \lambda_{i b} \dot{e}_{a}^{i}-\left(\Xi_{i}-\rho_{i}\right) \dot{\beta}^{i} \\
& -\left(\Theta_{i}-\omega_{i}\right) \dot{\alpha}^{i}-\left(\Sigma_{i}-\tau_{i}\right) \dot{\gamma}^{i}-\left(\Phi^{0}-\sigma^{0}\right) \dot{\varphi}_{0} .
\end{aligned}
$$

From the Lagrangian density (66) one may read off the final set of symplectic variables

$\xi^{I}=\left(A_{a}^{i}, \beta^{i}, e_{a}^{i}, \alpha^{i}, \lambda^{i}{ }_{a}, \gamma^{i}, \varphi^{0}, \rho^{i}, \omega^{i}, \tau^{i}, \sigma_{0}\right)$,

and the corresponding symplectic 1 -form is given by

$$
\begin{aligned}
a_{I}= & \left(\epsilon^{a b} \theta\left(\frac{1}{\mu} A_{b i}+2 e_{b i}\right),-\Xi_{i}+\rho_{i}, \epsilon^{a b} \lambda_{b i},-\Theta_{i}\right. \\
& \left.+\omega_{i}, 0,-\Sigma_{i}+\tau_{i},-\Phi_{0}+\sigma_{0}, 0,0,0,0\right) .
\end{aligned}
$$

After some algebra, we obtain the explicit form of the symplectic matrix $f_{I J}$ 


$$
\begin{gathered}
\left(\begin{array}{ccccccccccc}
2 \frac{\theta}{\mu} \eta_{i j} & -2 \frac{\theta}{\mu} \nabla_{a i j} & -2 \theta \eta_{i j} & -2 \theta \triangle_{a i j} & 0 & E_{a i j} & 0 & 0 & 0 & 0 & 0 \\
2 \frac{\theta}{\mu} \nabla_{a i j} & 0 & 2 \theta \triangle_{a i j} & 0 & -E_{a i j} & 0 & 0 & -\frac{1}{2} \epsilon_{a b} \eta_{i j} & 0 & 0 & 0 \\
2 \theta \eta_{i j} & -2 \theta \triangle_{a i j} & 0 & -2 \Lambda E_{a i j} & -\eta_{i j} & -D_{a i j}^{x} & -\lambda_{a j} & 0 & 0 & 0 & 0 \\
2 \theta \triangle_{a i j} & 0 & 2 \Lambda E_{a i j} & 0 & D_{a i j}^{y} & 0 & 0 & 0 & -\frac{1}{2} \epsilon_{a b} \eta_{i j} & 0 & 0 \\
0 & E_{a i j} & \eta_{i j} & -D_{a i j}^{x} & 0 & 0 & -e_{a j} & 0 & 0 & 0 & 0 \\
-E_{a i j} & 0 & D_{a i j}^{y} & 0 & 0 & 0 & 0 & 0 & 0 & -\frac{1}{2} \epsilon_{a b} \eta_{i j} & 0 \\
0 & 0 & \lambda_{a j} & 0 & e_{a j} & 0 & 0 & 0 & 0 & 0 & -\frac{1}{2} \epsilon_{a b} \\
0 & \frac{1}{2} \epsilon_{a b} \eta_{i j} & 0 & 0 & 0 & 0 & 0 & 0 & 0 & 0 & 0 \\
0 & 0 & 0 & \frac{1}{2} \epsilon_{a b} \eta_{i j} & 0 & 0 & 0 & 0 & 0 & 0 & 0 \\
0 & 0 & 0 & 0 & 0 & \frac{1}{2} \epsilon_{a b} \eta_{i j} & 0 & 0 & 0 & 0 & 0 \\
0 & 0 & 0 & 0 & 0 & 0 & \frac{1}{2} \epsilon_{a b} & 0 & 0 & 0 & 0
\end{array}\right) \\
\times \epsilon^{a b} \delta^{2}(x-y), \\
\end{gathered}
$$

such a matrix is not singular; the corresponding inverse matrix $f_{I J}{ }^{-1}$ is given by brackets given by (72)-(75). In addition, we are now ready to perform the counting of physical degrees of freedom: starting

$$
\begin{gathered}
\left(\begin{array}{ccccccccccc}
\frac{\mu}{2 \theta} \eta_{i j} & 0 & 0 & 0 & -\mu \eta_{i j} & 0 & 0 & D_{a i j}^{x} & -\frac{\mu}{2 \theta} L_{a i j} & -\frac{\mu}{2 \theta} E_{a i j} & \mu e_{a i} \\
0 & 0 & 0 & 0 & 0 & 0 & 0 & \eta_{i j} & 0 & 0 & 0 \\
0 & 0 & 0 & 0 & \eta_{i j} & 0 & 0 & -E_{a i j} & D_{a i j} & 0 & e_{a i} \\
0 & 0 & 0 & 0 & 0 & 0 & 0 & 0 & \eta_{i j} & 0 & 0 \\
\mu \eta_{i j} & 0 & -\eta_{i j} & 0 & -2 \theta \mu \eta_{i j} & 0 & 0 & L_{a i j} & -\vartheta_{a i j} & -\nabla_{a i j} & -\lambda_{a i} \\
0 & 0 & 0 & 0 & 0 & 0 & 0 & 0 & 0 & \eta_{i j} & 0 \\
0 & 0 & 0 & 0 & 0 & 0 & 0 & 0 & 0 & 0 & 1 \\
-D_{a i j}^{y} & -\eta_{i j} & E_{a i j} & 0 & -L_{a i j} & 0 & 0 & 0 & 0 & 0 & 0 \\
\frac{\mu}{2 \theta} L_{a i j} & 0 & -D_{a i j}^{y}-\eta_{i j} & \nabla_{a i j} & 0 & 0 & 0 & -\frac{\mu}{2 \theta} L_{a i k} L_{b}{ }^{k} j_{j} & 0 & 0 \\
\frac{\mu}{2 \theta} E_{a i j} & 0 & 0 & 0 & \nabla_{a i j} & -\eta_{i j} & 0 & 0 & 0 & -\frac{\mu}{2 \theta} E_{a i k} E_{b}{ }_{j}{ }_{j} e^{a j} \nabla_{a i j} \\
-\mu e_{a i} & 0 & -e_{a i} & 0 & \lambda_{a i} & 0 & -1 & 0 & 0 & -e^{a j} \nabla_{a i j} & 0
\end{array}\right) \\
\times \epsilon^{a b} \delta^{2}(x-y),
\end{gathered}
$$

with $\diamond_{a i j}=\left(\mu L_{a i j}+2 \Lambda E_{a i j}\right)$. In this way, the quantization bracket, dubbed generalized Faddeev-Jackiw bracket $\{,\}_{F-J}$ between two elements of the symplectic variable set (67), is defined as

$\left\{\xi_{I}(x), \xi_{J}(y)\right\}_{F-J} \equiv\left(f_{I J}\right)^{-1}$

The non-vanishing Faddeev-Jackiw brackets for topologically massive AdS gravity can now be easily extracted using (70) and (71);

$$
\begin{aligned}
\left\{A^{i}{ }_{a}(x), A^{j}{ }_{b}(y)\right\}_{F-J} & =\frac{\mu}{2 \theta} \eta^{i j} \delta^{2}(x-y), \\
\left\{A^{i}{ }_{a}(x), \lambda^{j}{ }_{b}(y)\right\}_{F-J} & =-\mu \epsilon_{a b} \eta^{i j} \delta^{2}(x-y), \\
\left\{\lambda^{i}{ }_{a}(x), \lambda^{j}{ }_{b}(y)\right\}_{F-J} & =2 \theta \mu \epsilon_{a b} \eta^{i j} \delta^{2}(x-y), \\
\left\{e^{i}{ }_{a}(x), \lambda^{j}{ }_{b}(y)\right\}_{F-J} & =\epsilon_{a b} \eta^{i j} \delta^{2}(x-y) .
\end{aligned}
$$

These F-J brackets correspond to the Dirac brackets reported in [32]. The canonical quantization $\left(\left\{\xi_{I}, \xi_{J}\right\}_{F-J} \rightarrow\right.$ $\left.\frac{1}{i \hbar}\left[\hat{\xi}_{I}, \hat{\xi}_{J}\right]\right)$ can be carried out by using the aforementioned with 18 canonical variables $\left(e_{a}^{i}, \lambda^{i}{ }_{a}, A^{i}{ }_{a}\right)$, we end up with 17 independent constraints $\left(\Xi_{i}^{(0)}, \Theta_{i}^{(0)}, \Sigma_{i}^{(0)}, \Phi^{0}, e^{i}=\right.$ $0, A^{i}{ }_{0}=0, \varphi_{0}=$ cte) after imposing the gauge-fixing term. Therefore, the number of physical degrees of freedom per space point for 3D Topologically Massive AdS Gravity is one, independently of the value of $\mu$, as it was also found in $[34,35]$.

\section{Conclusions and discussions}

In the present paper, the nature of the constraints and gauge structure of the topologically massive AdS gravity theory was studied from the perspective of the Faddeev-Jackiw symplectic approach. The whole set of independent physical constraints was identified through the consistency condition and the zero-modes. It was shown that even when all physical constraints are found, the symplectic matrix still has zero-modes, that is, when the zero-modes are orthogonal to the gradient of the symplectic potential on the surface of the constraints, 
one is led to deduce that the theory has a local gauge symmetry. Therefore, the zero-modes straightforwardly generate the local gauge symmetry under which all physical quantities are invariant. By mapping the gauge parameters appropriately we have also obtained the Poincaré transformations and the diffeomorphism symmetry. Additionally, we have shown that the time-gauge fixing of the density Lagrangian renders the non-degenerate symplectic matrix $f_{I J}$. We have then identified the quantizaion bracket (F-J brackets) structure and have proved that there is one physical degree of freedom. It is worth remarking that all the results presented here can be applied to the study of the physical content of models such as massive gravity and bigravity theories in $2+1$ dimensions, in which secondary, tertiary, or higher-order constraints are present. Such problems are under study and will be published elsewhere [57]. Another line for further research is the application of the procedure used here to explore conceptual and technical issues of gravity models in $3+1$ dimensions.

Acknowledgements This work has been partially supported by CONACyT under grand number CB-2014-01/240781. We would like to thank $\mathrm{G}$. Tavares-Velasco for reading a draft version of this paper and alerting us to various typos.

Open Access This article is distributed under the terms of the Creative Commons Attribution 4.0 International License (http://creativecomm ons.org/licenses/by/4.0/), which permits unrestricted use, distribution, and reproduction in any medium, provided you give appropriate credit to the original author(s) and the source, provide a link to the Creative Commons license, and indicate if changes were made.

Funded by SCOAP ${ }^{3}$.

\section{Appendix A: Faddeev-Jackiw symplectic approach}

In this appendix, we summarize the main aspects of the FJ symplectic approach [36], which is based on a first-order Lagrangian in the time derivative. However, this is not a serious restriction because even if the original Lagrangian is not of first-order, it is p ossible to introduce auxiliary fields in order to obtain a first-order one (usually, the canonical momenta are chosen as auxiliary fields see for instance the cites [53-55]). In this manner, we can construct a first-order Lagrangian for a physical system as follows

$\mathcal{L}(\xi)=a_{I}(\xi) \dot{\xi}^{I}-V(\xi) \quad(I=1,2,3, \ldots, N)$,

where $\xi^{I}$ are the so-called symplectic variables, which are identified as the set of dynamical variables of the theory. The term $V(\xi)$, which is called symplectic potential, is assumed to be free of time derivatives of $\xi^{I}$, and it is easy to see that in comparison with the Dirac method, the potential is the negative of the canonical Hamiltonian. Furthermore, the functions $a_{I}(\xi)$ are the canonical one-forms and they are of interest because they can be identified with either the original dynamical variables or the canonical momenta; in [FJ] framework there is a freedom for choosing the symplectic variables [53-55]. However, there are comments in this respect. First, if the momenta are introduced, then in the symplectic formalism they are not considered as primary constraints just like in Dirac's method is done. In the Faddeev-Jackiw formalism the momenta are part of the symplectic one-forms as auxiliar variables. Second, in order to obtain a symplectic tensor, the presence of the momenta force to fixing the gauge in a nontrivial form [54,55], however by using a suitable gauge, the Dirac and the generalized FJ brackets coincide to each other. Furthermore, the Euler-Lagrange equations of motion for Lagrangian (A1) can be written as

$f_{I J} \dot{\xi}^{J}-\frac{\partial}{\partial \xi_{I}} V(\xi)=0$,

where $f_{I J}$ is the so-called symplectic matrix with the following explicit form:

$f_{I J} \equiv \frac{\partial}{\partial \xi_{I}} a^{J}-\frac{\partial}{\partial \xi_{J}} a^{I}$.

If this matrix is non-singular, it can be inverted, and therefore the dynamical evolution of the symplectic variables can be solved from (A2)

$\dot{\xi}_{I}=(f)_{I J}^{-1} \frac{\partial}{\partial \xi_{J}} V(\xi)$.

Moreover, the equation (A4) can be written as

$\dot{\xi}_{I}=\left\{\xi_{I}, \xi_{J}\right\}_{F-J} \frac{\partial V}{\partial \xi_{J}}$.

where the Faddeev-Jackiw bracket $\{,\}_{F-J}$ is defined by

$\left\{\xi_{I}, \xi_{J}\right\}_{F-J}=(f)_{I J}^{-1}$

On the other hand, in gauge invariant theories, where in addition to the true dynamical degrees of freedom there are also gauge degrees of freedom, the symplectic matrix (A3) turns out to be singular, which implies that the system is endowed with constraints. In this case, the matrix $f_{I J}$ necessarily has some zero-modes $\left(v_{k}\right)$ (with $k$ all the linearly independent zero-modes that are found for $\left.f_{I J}\right)$, where each $\left(v_{k}\right)$ is a column vector with $N$ entries $\left(v_{k}\right)^{I}$. By definition, the zeromodes satisfy the following equation

$\left(v_{k}\right)^{I} f_{I J}=0, \quad(k=1,2,3, \ldots, \leq N)$.

Consequently, the constraints associated with the symplectic matrix are given by

$\phi_{k} \equiv\left(v_{k}\right)^{I} \frac{\partial}{\partial \xi^{I}} V(\xi)=0$, 
which shows that the zero-modes of $f_{I J}$ encode the information of the constraints. It is important to comment that if the momenta are introduced, then the constraints arising from (A8) coincide with the secondary constraints found in the Dirac method ( see the cites [37-41,53-55]). Following the prescription of the symplectic formalism, we will analyze if there are new constraints. Thus, for this aim, we impose a consistency condition on the constraints such as in the Dirac approach is used, namely

$\dot{\phi}_{k}=\frac{\partial \phi_{k}}{\partial \xi^{I}} \dot{\xi}^{I}=0$.

The consistency condition on the constraints (A9) and equations of motion (A2) can be rewritten as

$f_{K J}^{(1)} \dot{\xi}^{J}=Z_{K}^{(1)}(\xi)$,

where

$f_{K J}^{(1)}=\left(\begin{array}{c}f_{I J} \\ \frac{\partial \phi_{k}}{\partial \xi^{J}}\end{array}\right) \quad$ and $\quad Z_{K}^{(1)}(\xi)=\left(\begin{array}{c}\frac{\partial V}{\partial \xi^{I}} \\ 0\end{array}\right)$,

The new matrix $f_{I J}^{(1)}$ is not a square matrix anymore, however, it still contains linearly independent zero-modes $\left(v_{l}^{(1)}\right)$, which are different from those defined in (A7). Multiplying both sides of Eq. (A10) by these modes, we get the following constraint relations

$$
\left.\left(v^{(1)} l\right)^{K} Z_{K}^{(1)}\right|_{\phi_{k}=0}=0 .
$$

The substitution $\phi_{k}=0$ guarantees that these constraints will drop from the remainder of the calculation. If Eq. (A12) turn out to fulfill the identity $0=0$, then there are no further constraints; otherwise, the new constraints arising from Eq. (A12) are given by

$\left.\phi_{l}^{(1)} \equiv\left(v^{(1)} l\right)^{K} Z_{K}^{(1)}\right|_{\phi_{k}}=0$.

These new constraints can be treated in the same way as $\phi_{k}$. In other words, we can now introduce the consistency condition for $\phi_{l}^{(1)}$, as

$\dot{\phi}_{l}^{(1)}=\frac{\partial \phi_{l}^{(1)}}{\partial \xi^{I}} \dot{\xi}=0$.

and combine it with Eq. (A10) in order to construct a set of new linear equations, from which we explore whether there are more constraints. These steps are repeated until there are no further constraints in the system and the identities $0=0$ are fulfilled.

Once $m$ constraints are obtained through the consistency conditions of the constraints, we can modify our original Lagrangian (A1) by introducing the whole set of constraints multiplied by the corresponding Lagrangian multipliers $\dot{\eta}^{m}$ as follows:

$\mathcal{L}^{(E)}=a_{I}(\xi) \dot{\xi}^{I}+\phi_{m}(\xi) \dot{\eta}^{m}-V(\xi)^{(E)}$,

where $V(\xi)^{(E)}=\left.V(\xi)\right|_{\phi_{m}=0}$. We can now also calculate the new symplectic matrix associated with the modified Lagrangian, say $f_{I J}^{(E)}=\partial a_{J}^{(E)} / \partial \xi^{(E) I}-\partial a_{I}^{(E)} / \partial \xi^{(E) J}$ with $\xi^{(E) I}=\left(\xi^{I}, \eta^{l}\right)$; this new matrix can be either singular or non-singular. In the latter case it has an inverse and therefore all the new symplectic variables can be solved as in (A5); this scenario is equivalent to that present in the Hamiltonian formalism where there exist only second class constraints. In fact, in this scenario all the Lagrange multipliers can be found and the dynamics is determined. On the other hand, for gauge systems, the symplectic matrix is still singular and has no inverse unless some suitable gauge-fixing terms are introduced. In this way, the procedure can be finished and the Faddeev-Jackiw brackets can be identified as in (A6).

\section{References}

1. Supernova Search Team Collaboration, A.G. Riess et al., Observational evidence from supernovae for an accelerating universe and a cosmological constant. Astron. J. 116, 1009-1038 (1998)

2. R. Durrer, R. Maartens, Dark energy and dark gravity. Gen. Rel. Grav. 40, 301-328 (2008)

3. D. Lovelock, The Einstein tensor and its generalizations. J. Math. Phys. 12, 498501 (1971)

4. D. Lovelock, The four-dimensionality of space and the Einstein tensor. J. Math. Phys. 13, 874-867 (1972)

5. V.A. Rubakov, P.G. Tinyakov, Infrared-modified gravities and massive gravitons. Phys. Usp. 51, 759 (2008)

6. M. Fierz, W. Pauli, On relativistic wave equation for paticles of arbitrary spin in an electromagnetic field. Proc. R. Soc. Lond. A 173, 211-232 (1939)

7. C. de Rham, G. Gabadadze, Generalization of the Fierz-Pauli action. Phys. Rev. D 82, 044020 (2010)

8. C. de Rham, G. Gabadadze, A.J. Tolley, Resummation of massive gravity. Phys. Rev. Lett. 106, 231101 (2011)

9. C. de Rham, Massive gravity. Living Rev. Rel 17, 7 (2014)

10. S.F. Hassan, R.A. Rosen, Resolving the ghost problem in non-linear massive gravity. Phys. Rev. Lett. 108, 041101 (2012)

11. S.F. Hassan, R.A. Rosen, Confirmation of the secondary constraints and absense of ghost in massive gravity and bimetric gravity. JHEP 02, 026 (2012)

12. S.F. Hassan, R.A. Rosen, A. Schimidt-May, Ghost-free massive gravity with a general reference metric. JHEP 02, 126 (2012)

13. K. Hinterbichler, R.A. Rosen, Interacting Spin-2 Fields. JHEP 07, 047 (2012)

14. K. Hinterbichler, Theoretical aspects of massive gravity. Rev. Mod. Phys. 84, 671 (2012)

15. H.R. Afshar, E.A. Bergshoeff, W. Merbis, Interacting spin-2 fields in three dimensions. JHEP 01, 040 (2015)

16. C. Deffayet, J. Mourad, G. Zahariade, Covariant Constraints in ghost free massive gravity. JCAP 01, 032 (2013)

17. J. Kluson, Note about Hamiltonian structure of the non-linear massive gravity. JHEP 01, 013 (2012) 
18. K. Hinterbichler, R.A. Rosen, Interacting spin-2 fields. JHEP 07, 047 (2012)

19. D. Boulware, S. Deser, Can gravity have a finite range? Phys. Rev. D 6, 3368 (1972)

20. S. Deser, R. Jackiw, S. Templeton, Topologically massive gauge theories. Ann. Phys. 140, 372 (1982)

21. S. Deser, R. Jackiw, S. Templeton, Topologically massive gauge theories. Ann. Phys 281, 409 (2000)

22. S. Deser, R. Jackiw, S. Templeton, Topologically massive gauge theories. Ann. Phys 185, 406 (1988) (INSPIRE)

23. S. Deser, R. Jackiw, Three-dimensional Einstein gravity: Dynamical of flat space. Ann. Phys. (N. Y. ) 152, 220 (1984)

24. S. Deser, R. Jackiw, G. 't Hooft, Three-dimensional cosmological gravity: Dynamical of constant curvature. Ann. Phys. (N. Y. ) 153, 405 (1984)

25 . E. Witten, $(2+1)$-dimensional gravity as an exactly soluble system. Nucl. Phys. B 311, 46 (1988)

26. W. Li, W. Song, A. Strominger, Chiral gravity in three dimensions. JHEP 0804, 082 (2008)

27. S.S. Deser, R. Jackiw, S. Templeton, Three-Dimensional Massive Gauge Theories. Phys. Rev. Lett. 48, 975 (1982) (INSPIRE)

28. P.A.M. Dirac, Lectures Notes on Quantum Mechanics (Yeshiva University, New York, 1964)

29. T. Hanson, A. Egge, C. Teitelboim, Constraints Hamiltonian Systems (Accademia Nazionale dei Lincei, Roma, 1978)

30. M. Henneaux, C. Teitelboim, Quantization of Gauge Systems (Princeton University Press, Princeton, 1991)

31. L. Castellani, Symmetries in the contrained Hamiltonian system. Ann. Phys. 143, 357 (1982)

32. M. Blagojevic, B. Cvetkovic, Canonical structure of topologically massive gravity with a cosmological constant. JHEP 05, 073 (2009)

33. M.-I. Park, Constraint dynamics and gravitons in three dimensions. JHEP 0809, 084 (2008)

34. S. Carlip, Constraint algebra of topologically massive AdS gravity. JHEP 0810, 078 (2008)

35. D. Grumiller, R. Jackiw, N. Johansson, Canonical analysis of cosmological topologically massive gravity at the chiral point, MITCTP 3957, UUITP-12/08 (2008)

36. L.D. Faddeev, R. Jackiw, Hamiltonian reduction of unconstrained and constrained systems. Phys. Rev. Lett 60, 1692 (1988)

37. J. Barcelos-Neto et al., Symplectic quantization of constrained systems. Mod. Phys. Lett. A 7, 1737 (1992)

38. J. Barcelos-Neto, C. Wotzasek, Faddeev-Jackiw quantization and constraints. Int. J. Mod. Phys. A 7, 4981 (1992)

39. H. Montani, R. Montemayor, Lagrangian approach to a symplectic formalism for singular systems. Phys. Rev. D 58, 125018 (1998)
40. H. Montani, C. Wotzasek, Faddeev-Jackiw quantization of nonabelian systems. Mod. Phys. Lett. A 8, 3387 (1993)

41. J.A. Garcia, J.M. Pons, Equivalence of Faddeev-Jackiw and Dirac approaches for gauge theories. Int. J. Mod. Phys. A 12, 451 (1997)

42. L. Liao, Y.C. Huang, Non-equivalence of Faddeev-Jackiw method and Dirac-Bergmann algorithm and the modification of FaddeevJackiw method for keeping the equivalence. Ann. Phys. 322, 2469 (2007)

43. E.M.C. Abreu, A.C.R. Mendes, C. Neves, W. Oliveira, R.C.N Silva, C. Wotzasek, Obtaining non-Abelian field theories via the Faddeev-Jackiw symplectic formalism. Phys. Lett. A 375, 3603 (2010)

44. C. Wotzasek, Faddeev-Jackiw approach to hidden symmetries. Ann. Phys. 243, 73 (1995)

45. S. Carlip, Inducing Liouville theory from topologically massive gravity. Nucl. Phys. B 362, 111-124 (1991)

46. S. Carlip, S. Deser, A. Waldron, D.K. Wise, Cosmological topologically massive Gravitons and Photons, class. Quantum Grav. 26, 075008 (2009)

47. R. Utiyama, Invariant theoretical interpretation of interaction. Phys. Rev. 101, 1597 (1956)

48. T.W.B. Kibble, Lorentz invariance and the gravitational field. J. Math. Phys 2, 212 (1961)

49. R. Banerjee, S. Gangopadhyay, P. Mukherjee, D. Roy, Symmetries of the general topologically massive gravity in the Hamiltonian and Lagrangian formalisms. JHEP 1002, 075 (2010)

50. M. Blagojevic, Gravitation and Gauge Symmetries (IOP, Bristol, 2002)

51. D.J. Toms, Faddeev-Jackiw quantization and the path integral. Phys. Rev D 92, 105026 (2015)

52. A. Fuster, Marc Henneaux and Axel. Maas, BRST quantization: A short review. Int. J. Geom. Meth. Mod. Phys. 2, 939-964 (2005)

53. Y. Jin-Long, H. Yong-Chang, Improved Faddeev-Jackiw quantization of the electromagnetic field and Lagrange multiplier fields. Chin. Phys. C 32(10), 788-792 (2008)

54. A. Escalante, J. Manuel-Cabrera, Hamiltonian dynamics and Faddeev-Jackiw formulation of 3D gravity with a BarberoImmirzi like parameter. Eur. Phys. J. C 77, 303 (2017)

55. A. Escalante, J. Manuel-Cabrera, Faddeev-Jackiw quantization of an Abelian and non-Abelian exotic action for gravity in three dimensions. Ann. Phys. 361, 585-604 (2015)

56. A. Escalante, O. Rodríguez-Tzompantzi, Hamiltonian dynamics and gauge symmetry for three-dimensional Palatini theory with cosmological constant. JHEP 05, 073 (2014)

57. Omar Rodríguez-Tzompantzi (2018) (under preparation) 